The output mode cleaner of GEO 600

This article has been downloaded from IOPscience. Please scroll down to see the full text article.

2012 Class. Quantum Grav. 29055009

(http://iopscience.iop.org/0264-9381/29/5/055009)

View the table of contents for this issue, or go to the journal homepage for more

Download details:

IP Address: 194.94.224.254

The article was downloaded on 25/03/2013 at 11:02

Please note that terms and conditions apply. 


\title{
The output mode cleaner of GEO 600
}

\author{
M Prijatelj ${ }^{1}$, J Degallaix ${ }^{2}$, H Grote $^{1}$, J Leong $^{1}$, C Affeldt $^{1}$, S Hild $^{3}$, \\ H Lück $^{1}$, J Slutsky ${ }^{1}$, H Wittel $^{1}$, K Strain $^{1,4}$ and K Danzmann $^{1}$ \\ ${ }^{1}$ Max-Planck-Institut für Gravitationsphysik (Albert-Einstein-Institut), \\ D-30167 Hannover, Germany \\ 2 Laboratoire des Matériaux Avancés, 69600 Villeurbanne, France \\ ${ }^{3}$ School of Physics and Astronomy, University of Birmingham, Edgbaston, \\ Birmingham B15 2TT, UK \\ ${ }^{4}$ Institute for Gravitational Research, University of Glasgow, Glasgow G12 8QQ, UK \\ E-mail: mirko.prijatelj@aei.mpg.de
}

Received 13 November 2011, in final form 18 January 2012

Published 14 February 2012

Online at stacks.iop.org/CQG/29/055009

\begin{abstract}
The German-British interferometric gravitational wave detector GEO 600 is currently undergoing upgrades within the GEO-HF upgrade program. The goal of this program is to enhance the sensitivity of GEO 600, in particular at frequencies above $500 \mathrm{~Hz}$. At these frequencies, the detector is limited by shot noise. An important element of the upgrade is the implementation of an output mode cleaner (OMC). This filtering cavity suppresses higher order spatial and temporal modes in the interferometer's output beam, thereby reducing the shot noise and enhancing sensitivity. Fully automated lock acquisition for the OMC was developed in order to ensure a high duty cycle of GEO 600. To maintain optimum sensitivity, the resonance condition of the OMC must be matched to the output beam from the interferometer. This requires continuous control of the resonance frequency of the OMC to the light, and alignment of the beam to the OMC. We describe the design and implementation of the OMC with special attention to the control techniques employed. We present results from the experience gained during the S6/VSR3 science run. Furthermore, we describe an upper limit measurement of the internal displacement noise of a piezoelectric actuator.
\end{abstract}

PACS numbers: 04.80.Nn, 07.60.Ly

(Some figures may appear in colour only in the online journal)

\section{Introduction}

The GEO-HF program, which is an upgrade to the GEO 600 gravitational wave detector, is currently underway. The main goal of this program is to enhance the sensitivity at frequencies 
where the detector is limited by shot noise, i.e. above $500 \mathrm{~Hz}$. Some improvement at lower frequencies is to be expected.

The program, which was started in 2009 , consists of a number of steps each of which is designed to enhance the shot noise limited sensitivity either by increasing the signal size at high frequency or by reducing the detection noise. In time order, these steps are: a change in the detection scheme from heterodyne readout to homodyne/dc readout [1], the implementation of an output mode cleaner (OMC), the increase of the signal recycling bandwidth and the implementation of squeezing. The final step, planned to start later this year, is to increase the circulating light power by employing a more powerful laser and modifying the injection optics and other interferometer systems to handle increased power [2,3].

The implementation of an OMC improves the shot-noise-limited sensitivity by reducing the detection noise. In dc readout, the $\mathrm{TEM}_{00}$ carrier light leaving the interferometer due to an intentional arm length difference (dark-fringe offset) needs to dominate the other components of the output beam, such as higher order optical modes and sideband fields, that do not contribute to the signal but increase the shot noise, to approach the optimum signal to noise ratio. Reduction of higher order modes and sideband fields allows for a smaller dark-fringe offset. This, in turn, reduces coupling coefficients for several noise sources, including laser amplitude noise, and also increases the interferometer's stability.

The challenge in implementing an OMC is ensuring that it provides continuous, nearoptimal transmission of the $\mathrm{TEM}_{00}$ carrier light and the signal light, which shares the same mode. Static errors in this reduce the sensitivity via reduction of transmitted carrier light and also increase noise couplings. Dynamic errors constitute new noise sources [4]. To keep the optimum transmission, the resonance frequency of the OMC and the frequency of the interferometer output beam must be kept identical and the alignment of the output beam onto the OMC must be kept optimal.

In section 2, we detail the design of the OMC and its implementation into GEO 600. We also describe the control systems for the OMC frequency and beam alignment. In section 3 , we discuss the robustness and performance of the OMC as experienced during the S6/VSR3 science run. In section 4, we establish upper limits for the internal displacement noise of a multi-stack piezoelectric actuator (PZT). In these measurements, the interferometer's output beam serves as an ultra-stable light source. We then summarize our findings and discuss the outlook of future work.

\section{Implementation of the OMC}

\subsection{Optical specifications and design of the OMC}

The OMC is an essential part of the GEO 600 interferometer, particularly now that it is operated in dc readout. Without the OMC, the full benefit of using the dc readout control technique could not be reached. The OMC is designed to filter the beam spatially, to remove higher order modes and, temporally, to remove unwanted frequency components, such as $15 \mathrm{MHz}$ control sideband fields ${ }^{5}$.

The specifications for the OMC were derived by setting an upper limit for the shot noise contribution from the sidebands and the higher order optical modes. At the beginning of GEO-HF, it was decided to use a small dark-fringe offset (around $20 \mathrm{pm}$ ) to increase the interferometer stability and allows the detection to be achieved with only one photodiode even

5 Even if the $15 \mathrm{MHz}$ sidebands are no longer required to derive the error signal to keep the interferometer on the dark fringe, they are still necessary for the interferometer's alignment control system which is then implemented before the OMC. 


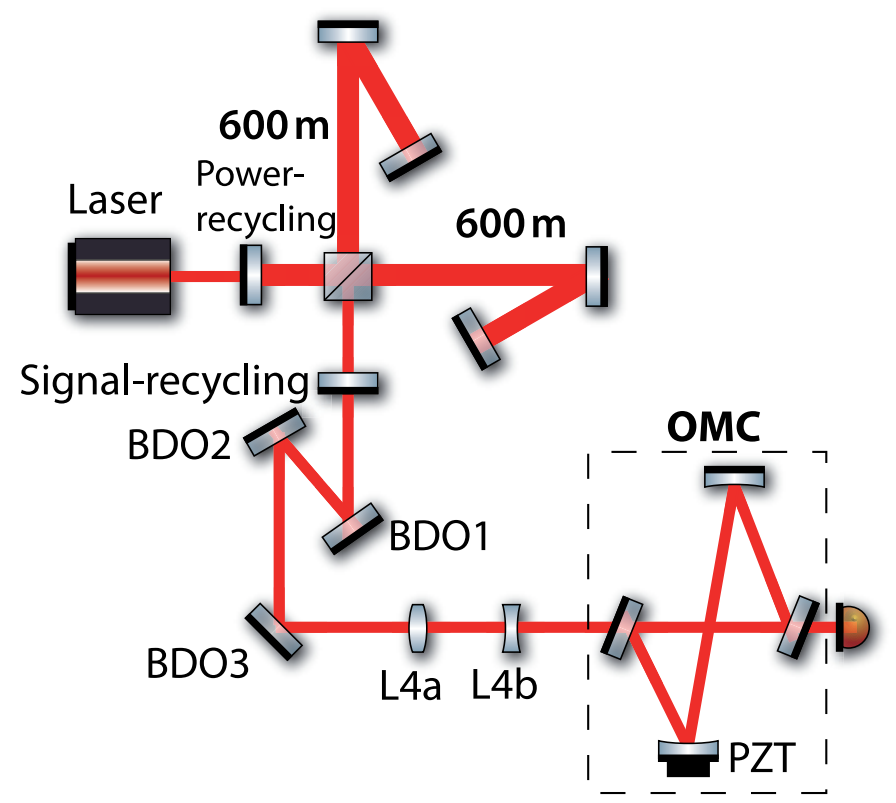

Figure 1. Simplified GEO 600 layout (not up to scale, OMC magnified for clarity).

after the future ten times increase in the circulating power. The dark-fringe offset of $20 \mathrm{pm}$ results in $4 \mathrm{~mW}$ of optical power used as the local oscillator for the detection at the output of GEO. For the sidebands and the higher order modes to not increase the measured shot noise level by more than $1 \%$, the combined power of these two fields must be less than $0.4 \mathrm{~mW}$.

From preliminary experiments in preparation of the dc-readout configuration, it was shown that the optical power at the output could be considered as equally partitioned between sidebands and higher order modes and that the optical power at the output was around $40 \mathrm{~mW}$. As such, the following two main specifications were derived.

(i) The $15 \mathrm{MHz}$ sideband's power must be attenuated by at least a factor 100 in power in transmission of the OMC. This requirement produces a lower limit on the bandwidth of OMC optical cavity.

(ii) The higher order optical mode's power must also be attenuated by at least a factor 100 in transmission. This requirement gives a lower limit on the cavity finesse and also restricts the choice of the possible $g$-factor for the OMC.

The above specifications were set mindful that GEO 600 will later use a $35 \mathrm{~W}$ input laser.

To ensure that the $\mathrm{OMC}$ meets these specifications, simulation tools were developed based mainly on analytical formulas and $\mathrm{ABCD}$ propagation laws. Then, the optimal parameters found were later checked with the Finesse software [5].

During the simulations, it was decided that the OMC will consist of a four-mirror cavity in diamond shape as seen in the dashed box in figure 1. This particular configuration features several advantages. First, it can be made compact in the direction of the beam, which suited the conditions found at the GEO site. Second, the center of mass of the structure is along the optical axis defined by the input and output beams. The equal distribution of mass on either side of the optical axis simplifies the seismic isolation. Third, of the four mirrors of the cavity, two are used as input and output mirrors, one is mounted on a PZT for length control. Finally 


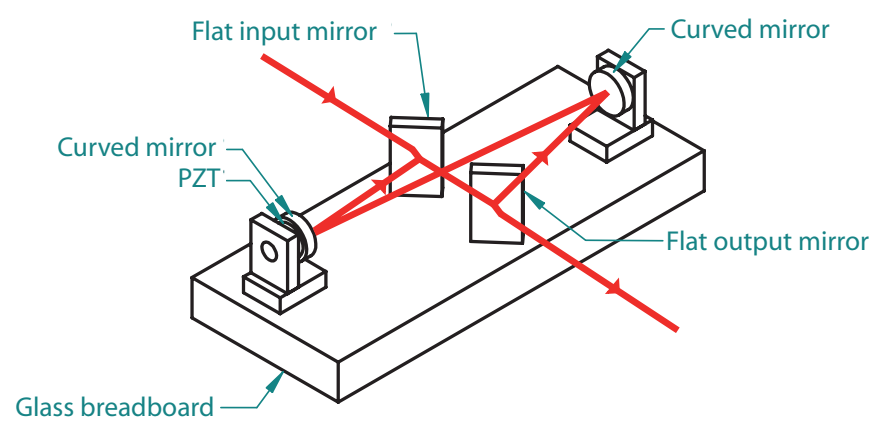

Figure 2. CAD view of the GEO 600 OMC. All parts are made of fused silica except for the PZT. The glass breadboard is $400 \mathrm{~mm} \times 160 \mathrm{~mm}$ and $38 \mathrm{~mm}$ thick.

the remaining mirror can be partially transparent and be used as a pickup to monitor the beam circulating in the OMC. And last but not least, using an even number of mirrors kept the resonance degeneracy between the transverse electro-magnetic modes of orders $m, n$ and $n, m$. So, when scanning through one free spectral range of the OMC the modes $\mathrm{TEM}_{10}$ and $\mathrm{TEM}_{01}$ appear combined in a single-transmission peak.

We decided on a finesse of 155, a $g$-factor of 0.73 and a round trip length of $66 \mathrm{~cm}$ according to the simulations. The size of the laser beam waist in the OMC is then $440 \mu \mathrm{m}$.

For thermal stability, the OMC is entirely made of fused silica glass with optics and mounts glued with UV-cured epoxy. A CAD view of the OMC can be found in figure 2. More details of the design and the construction of the OMC can be found in [6].

\subsection{Implementation in $G E O$}

The original GEO 600 design called for heterodyne readout and a very small OMC. To be able to install this, much larger, OMC necessary for dc readout at the output of GEO 600, a new vacuum tank had to be incorporated in the existing GEO 600 infrastructure. This tank was not only necessary for the OMC, but could also house a large Faraday isolator, as well as the detection photodiode. This new tank has a dedicated vacuum pump, and a septum window separates it from the main vacuum system allowing rapid access to the OMC without having to vent the entire system. The new tank was installed in GEO during the summer 2009 and the OMC was placed in vacuum in December 2009.

The OMC is isolated from the motion of the vacuum tank. A $700 \mathrm{~mm} \times 700 \mathrm{~mm}$ metal plate is isolated from the bottom of the tank by sitting it on five Viton O-rings. On this plate, there are 3 Minus K SM-30 isolators. These provide isolation from motion of $10 \mathrm{~dB}$ at $1 \mathrm{~Hz}$ and $45 \mathrm{~dB}$ above $10 \mathrm{~Hz}$ in the horizontal and vertical directions, respectively. They in turn support a $700 \mathrm{~mm} \times 700 \mathrm{~mm}$ custom-made breadboard that is $8 \mathrm{~mm}$ thick. After initial tests Viton pads were placed between the OMC and the breadboard adding further isolation above $50 \mathrm{~Hz}$ in the horizontal and vertical directions reducing sideband structures around the OMC length dither at $6 \mathrm{kHz}$ (see section 2.4).

The mode matching between the output beam of the interferometer and the OMC is made more complex than usual due to the limited amount of space available and the high higher order mode content of the GEO 600 output beam. To keep the same suspended mirrors (the mirrors called BDO1, 2, 3 in figure 1) used for beam directing and focusing, it was decided to add two lenses in front of the OMC. The lenses were mounted on the same breadboard as the 
OMC and the mode matching can be optimized by translating these lenses. The high content of higher order modes in the carrier light can disturb the assessment of the mode matching. We therefore used the spatially cleaner $15 \mathrm{MHz}$ sidebands to assess the mode matching. We achieved a mode-matching efficacy of approximately $90 \%$ for these sidebands and assume the mode matching for the carrier light to be similar.

\subsection{Control setup}

We had to implement control systems for the OMC resonant frequency by adjusting its length, and for the beam alignment by feeding back to angles of the beam-director mirrors. We call the former the longitudinal control, and the latter the alignment control. We decided to use dither locking [7] to generate error signals for both controls, because this technique does not require additional new hardware beyond the control system itself. Earlier simulations [4] suggested that rather low bandwidth control systems could be employed for longitudinal and alignment control.

The OMC control is the first application of the fully digital control and data (CDS) [8] system in GEO 600. It is based on, and very similar to, the system planned for Advanced LIGO. Most pre-existing GEO control systems are analog electronics, supervised by National Instruments LabView (LV) 6.1. The OMC control systems needed to be interfaced with preexisting GEO 600 control systems. A LV 2009 PC interfaces the CDS with pre-existing LV 6.1. LV 2009 communicates with CDS via channel access protocol and with LV 6.1 via direct UDP link at $10 \mathrm{~Hz}$. Furthermore, LV 2009 is used for some high-level OMC controls.

For GEO 600 to achieve a high duty cycle, it is necessary that all its subsystems are highly automated. This necessitates an automated lock acquisition for the OMC. During this, the differential arm length of the interferometer is controlled via an auxiliary photodiode in transmission of BDO1. The acquisition algorithm is implemented in C-code in CDS. Its required complexity depends on the mode content of the interferometer's output beam. We chose the dc readout configuration with tuned signal recycling, reduced control sideband intensity and intentional dark-fringe offset for lock acquisition. In this configuration, the TEM $\mathrm{T}_{00}$ carrier light is the strongest component of the output beam (see figure 3 ). The algorithm scans across one free spectral range and records the maximum transmitted power. It then repeats the scan and stops at the previously marked position and activates the longitudinal control of the OMC. Control of the Michelson interferometer arm length difference is then transferred to the photodiode in transmission of the OMC. Use of this less noisy photodiode reduces the overall noise level in the control loop controlling the difference in the arm length. Only these lower noise levels allow us to activate the OMC alignment control.

\subsection{Longitudinal control}

A PZT (Physik Instrumente S-303) is used for controlling the length of the OMC. The PZT actuation range corresponds to approximately six free spectral ranges of the OMC. The PZT dithers the OMC length at $6 \mathrm{kHz}$ with a peak-to-peak amplitude of about $1 \mathrm{pm}$. We decided on a unity gain frequency (UGF) for longitudinal control of $8 \mathrm{~Hz}$ and a de gain of $92 \mathrm{~dB}$. A higher UGF of $80 \mathrm{~Hz}$ showed no apparent benefits.

\subsection{Alignment control}

The BDO1 and BDO3 mirrors are used to control the alignment. They are dithered in rotation in the horizontal plane and the vertical plane (in tilt) at the frequencies shown 


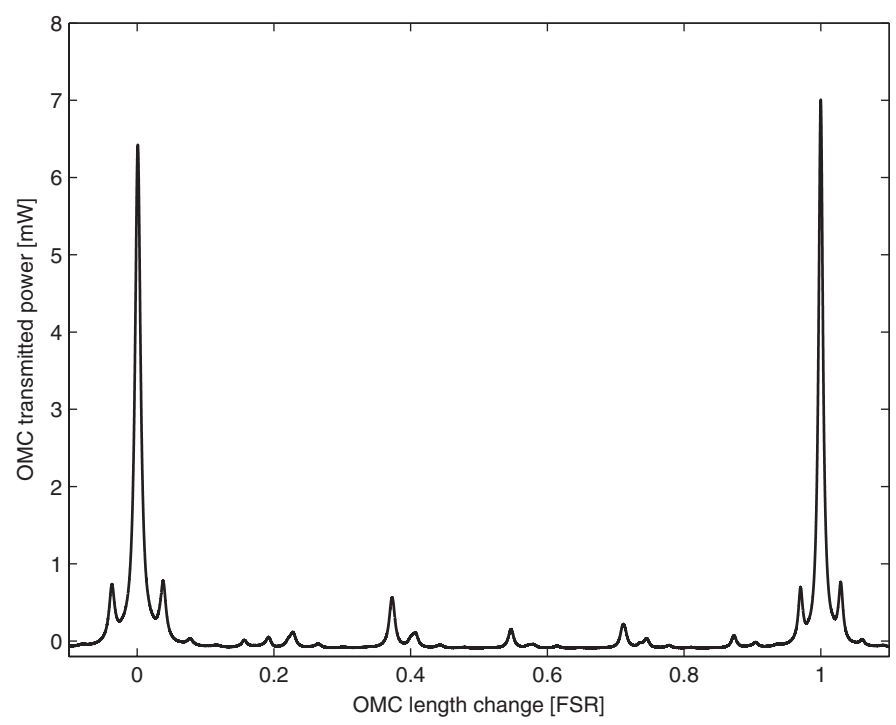

Figure 3. A mode scan of the OMC over one free spectral range. The tall spikes are $\mathrm{TEM}_{00}$ carrier light, mostly caused by the intentional arm length difference of the interferometer. Adjacent to these with a magnitude of approximately $0.8 \mathrm{~mW}$ are control sidebands at $14.9 \mathrm{MHz}$. The other elements are higher order optical modes.

Table 1. Alignment control loop gain hierarchy as employed during S6/VSR3.

\begin{tabular}{lccc}
\hline Degree of freedom & Dither frequency $(\mathrm{Hz})$ & Unity gain frequency $(\mathrm{Hz})$ & DC gain $(\mathrm{dB})$ \\
\hline BDO1 rotation & 265 & 0.024 & 16 \\
BDO1 tilt & 420 & 0.022 & 15 \\
BDO3 rotation & 530 & 0.011 & 7 \\
BDO3 tilt & 480 & 0.040 & 25 \\
\hline
\end{tabular}

in table 1 . We chose frequencies at which the GEO 600 sensitivity was best, giving the optimum signal to noise ratio for the alignment control. We implemented a gain hierarchy (see table 1) with one mirror dominating in rotation and tilt, respectively. The highest UGFs were at $0.024 \mathrm{~Hz}$ in rotation and $0.040 \mathrm{~Hz}$ in tilt. This is well below the resonance frequencies of $\mathrm{BDO} 1$ and $\mathrm{BDO} 3$ at about $1 \mathrm{~Hz}$. DC gains were $16 \mathrm{~dB}$ and $25 \mathrm{~dB}$, respectively.

\section{Long term operation of the OMC subsystem}

We were able to collect extensive experience with the new GEO 600 configuration during the S6/VSR3 joint science run with the LIGO and VIRGO detectors from 11 August to 20 October 2010. GEO 600 achieved a science data duty factor of $88 \%$. The longest lock exceeded $62 \mathrm{~h}$. The OMC lock acquisition had a success rate well above 90\%. The OMC subsystem ran autonomously for weeks at a time.

We quantified the noise contributions of frequency and alignment control systems via noise projections [10]. We projected their feedback signals to strain sensitivity (see figure 4). The OMC control feedback noise is well below the overall GEO 600 sensitivity. These measurements were performed after the exchange of the $2 \%$ transmission 


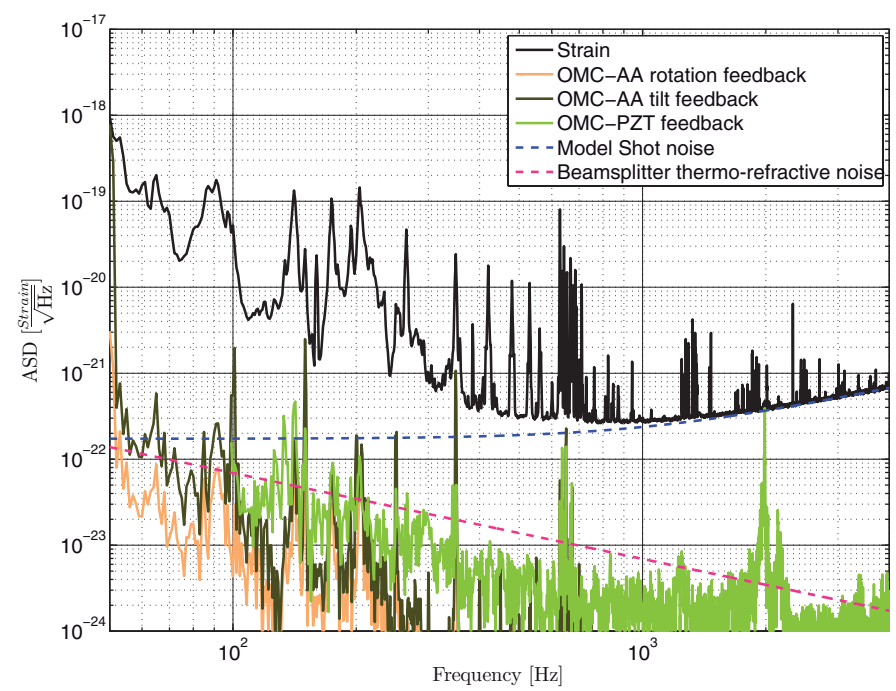

Figure 4. Noise contributions from OMC control feedbacks. Note that the alignment noise curves are strictly upper limits. These measurements were performed after the exchange of the $2 \%$ transmission signal-recycling mirror for a $10 \%$ signal-recycling mirror. Details regarding thermorefractive noise can be found in [9].

signal-recycling mirror for a $10 \%$ signal-recycling mirror [2]. This increased the higher order mode content in the GEO output beam, which should be negligible for these measurements.

\section{Measurements of an upper limit of the internal displacement noise of a PZT}

Looking at the interferometer's output beam as an ultra-stable light source gives us the opportunity to measure an upper limit of the internal displacement noise of the Physik Instrumente S-303 PZT OMC length actuator.

We locked the OMC to a transmitted power of $90 \%$ of the maximum achieved TEM $_{00}$ carrier light transmission. At this setting, off of peak transmission, fluctuations in the OMC length couple directly to fluctuations in transmitted power. This increased power noise in the transmitted beam (see figure 5). We calculated the coupling coefficient from length fluctuations $\left(\mathrm{m} \mathrm{Hz}^{-1 / 2}\right)$ to fluctuations in light power $\left(\mathrm{W} \mathrm{Hz}^{-1 / 2}\right)$ for $6 \mathrm{~mW}$ transmitted power using Finesse simulations to $1.78 \times 10^{6} \mathrm{~W} \mathrm{~m}^{-1}$. We arrive at an upper limit for OMC length fluctuations if we attribute all noise in the OMC transmitted power to OMC length fluctuations. As noise projections show some of this noise can be attributed to sources other than PZT internal displacement noise. Such noise sources are OMC length fluctuations due to electric noise of the PZT HV amplifier output, fluctuations of the laser amplitude, fluctuations of the interferometers output frequency due to length fluctuations of the power-recycling cavity [11] and shot noise. The peak at $6 \mathrm{kHz}$ is due to the OMC length dither. It was beyond the scope of this paper to investigate the origin of the other remaining noise peaks. We achieve an upper limit of approximately $2 \times 10^{-14} \mathrm{~m} \mathrm{~Hz}^{-1 / 2}$ (see figure 6) for broadband PZT internal displacement noise after subtracting the incoherent sum of the known noise sources. Earlier measurements either reached worse sensitivities in the $1 \times 10^{-11} \mathrm{~m} \mathrm{~Hz}^{-1 / 2}$ range $[12,13]$, 


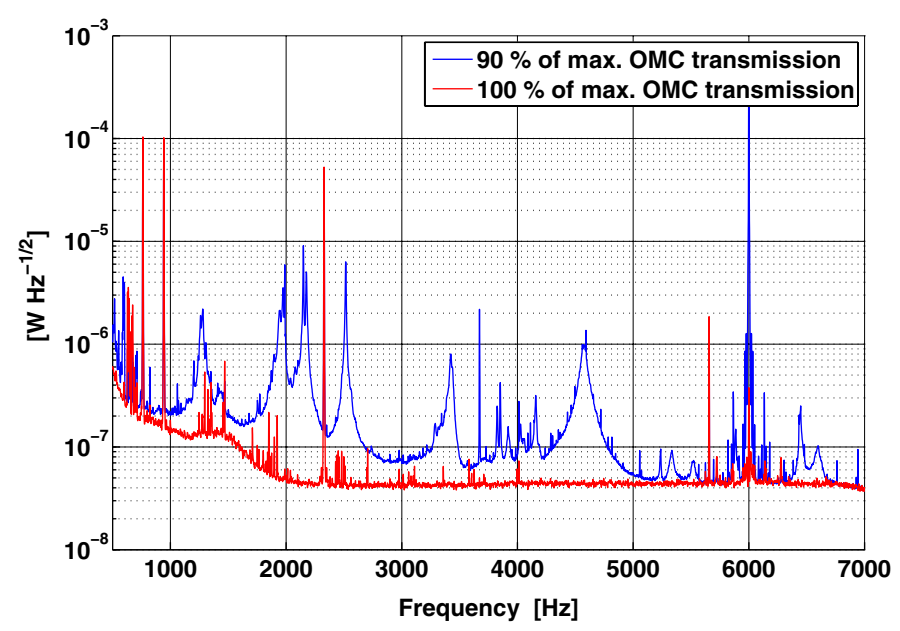

Figure 5. Power fluctuations in the OMC transmitted beam for different transmissions.

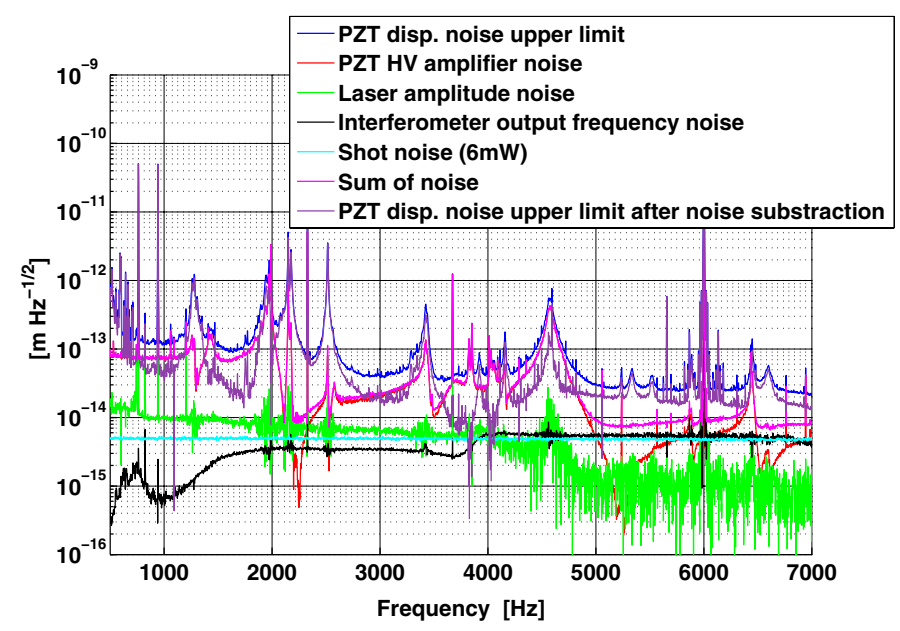

Figure 6. Projection of obvious sources contributing to noise in OMC transmitted power, and upper limit for PZT internal displacement noise before and after noise subtraction.

or were limited by their nature as in-loop measurements [14]. A similar sensitivity was reached in [15].

\section{Conclusion and outlook}

The OMC has proven to be a reliable, well-performing subsystem. Due to its autonomous mode of operation, it adds minimal extra complexity to the day-to-day operation of GEO 600. We established an upper limit on the internal displacement noise of a wide range PZT utilizing the OMC. 


\section{Acknowledgments}

We acknowledge the GEO collaboration for their ongoing support of GEO 600. The Max Planck Society, the Science and Technology Facilities Council in the UK, the Bundesministerium für Bildung und Forschung and the State of Lower Saxony in Germany, and the Volkswagen Foundation all made generous contributions which helped to make GEO 600 a reality. Many people over many years have also contributed hard work and dedication to the design and construction, as well as upgrades to GEO 600 that make it what it is today. We thank the Albert-Einstein-Institut, Hannover, and the University of Glasgow, UK, for providing support and a stimulating environment from which the ideas that led to this work grew.

\section{References}

[1] Hild S et al 2009 Class. Quantum Grav. 26055012

[2] Wilke B et al 2006 Class. Quantum Grav. 23 S207

[3] Grote H (for the LIGO Scientific collaboration) 2010 Class. Quantum Grav. 27084003

[4] Prijatelj M et al 2010 J. Phys.: Conf. Ser. 228012014

[5] Freise A, Heinzel G, Lück H, Schilling R, Willke B and Danzmann K 2003 Class. Quantum Grav. 21 1067-74

[6] Degallaix J et al 2010 J. Phys.: Conf. Ser. 228012013

[7] Gascoyne P F and Rutt H N 1983 J. Phys. E: Sci. Instrum. 1631

[8] Bork R 2010 LIGO Technical Report T0900612 (https://dcc.ligo-.org/cgi-bin/DocDB/ShowDocument?.submit=Number\&docid=T0900612\&version=)

[9] Benthem B and Levin Y 2009 Phys. Rev. D 80062004

[10] Smith J R, Ajith P, Grote H, Hewitson M, Hild S, Lück H, Strain K A, Wilke B, Hough J and Danzmann K 2006 Class. Quantum Grav. 23 527-37

[11] Wilke B et al 2002 Class. Quantum Grav. 19 1377-87

[12] Bergamin A, Cavagnero G, Cordiali L, Mana G and Zosi G 1997 IEEE Trans. Instrum. Meas. 46576

[13] Aplin K L and Middleton K F 2007 J. Phys.: Conf. Ser. 85012013

[14] Gray M B, McClelland D E, Barton M and Kawamura S 1999 Opt. Quantum Electron. 31 571-82

[15] Pisani M 2009 Meas. Sci. Technol. 20084008 